\title{
Incretin Based Therapeutics: Connections to Vascular Biology and Implications for Potential Cardiovascular Disease Prevention
}

\author{
Editorial to: "Glucagon-like Peptide-1 Receptor Agonist Liraglutide Inhibits Endothelin-1 \\ in Endothelial Cell by Repressing Nuclear Factor-Kappa B Activation” by Dai et al.
}

\author{
Anthony E. Dear
}

Published online: 31 May 2013

(C) Springer Science+Business Media New York 2013

The incretin Glucagon like peptide-1 (GLP-1) has a well documented role in glucose-stimulated insulin release and the preservation of plasma glucose homeostasis [1]. The importance of the incretin system in glucose homeostasis is emphasised by the recent successful therapeutic exploitation of this system utilising GLP-1 receptor (GLP-1R) agonists and inhibitors of the dipeptidyl peptidase 4 (DPP-4) enzyme in the treatment of type 2 diabetes [2].

Identification of the GLP-1R in tissues outside the pancreatic $\beta$ cell and in particular on vascular endothelial cells [3] suggests a role for GLP-1 in vascular biology. In vitro and in vivo studies demonstrate that native GLP-1 and GLP-1R agonists regulate vascular tone and endothelial function [4-8], and clinical studies have reported reduced endothelial dysfunction in type 2 diabetic patients with established coronary artery disease [3]. Several in vivo studies identify atheroprotection by native GLP-1, GLP-1R agonists and DPP-4 inhibitors [9-12]. The potential athero-protective effects of glucagon-like peptide-1 (GLP-1), long-acting GLP-1 analogues and GLP-1R agonists are currently the subject of intense pre-clinical and clinical research given type 2 diabetes is characterized by accelerated atherosclerosis [13].

Earlier clinical trials with liraglutide, an acylated GLP-1R agonist, demonstrated improved levels of both fasting and postprandial glucose, improvement in $\beta$-cell function, a reduction in plasma glucagon concentration, weight loss, reduced blood pressure and an improvement in cardiovascular risk biomarkers in patients with type 2 diabetes [14-17].

\footnotetext{
A. E. Dear $(\bowtie)$

Eastern Clinical Research Unit: Translational Research Division, Department of Medicine, Monash University, Level 2, 5 Arnold Street, Box Hill 3128, Melbourne, Australia e-mail: anthony.dear@monash.edu
}

Liraglutide attenuates endothelial cell dysfunction, a known predictor of cardiovascular events, together with in vivo expression of vascular adhesion molecules, atherogenesis and enhances plaque stability and is currently the subject of a clinical trial programme to evaluate safety and efficacy in cardiovascular disease prevention $[8,18,19]$.

Whilst a mounting body of credible scientific evidence supports a potential role for the incretin system in general and GLP-1R agonists including liraglutide specifically in the modulation of the atherogenesis process the presence of recent, convincing however discordant publications forces a reevaluation of the underlying molecular mechanisms which incretin based therapeutics may be engaging in the vasculature in order to modulate potential disease processes [20]. The study by Dai and colleagues in this issue of Cardiovascular Drugs and Therapy adds significantly to our understanding at the cellular and molecular level of the mechanisms possibly responsible for incretin based therapeutic activity in the vasculature and potential contribution to atheroprotection [21]. Dai and co-workers, using a human vascular endothelial cell model, present in vitro evidence identifying liraglutide-mediated modulation of molecules central to vascular endothelial integrity together with cellular localisation studies and identify a potential molecular mechanism responsible for this effect [21]. The differential effects of liraglutide on basal and Phorbol Ester (PMA) and Tumour Necrosis Factor- $\alpha$ (TNF $\alpha)$-mediated endothelial nitric oxide synthase (eNOS) and endothelin-1 (ET-1) expression is accompanied by modulation of NFKB and I $k \mathrm{~B} \alpha$ phosphorylation status together with inhibition of induced proinflammatory cytokine interleukin-6 (IL-6) expression all within the same cell system, under hyperglycaemic conditions and at concentrations of liraglutide approximating those identified in vivo [22]. Whilst these in vitro results support previous observations identifying liraglutide- 
mediated regulation of endothelial function $[8,18]$, and NFKB activity [23] their strength lies in performing the experiments under hyperglycaemic conditions and observing differential regulation of two molecules, eNOS and ET-1, critical to vascular endothelial integrity, simultaneously. In addition the intriguing effect of liraglutide-mediated regulation of GLP-1R expression also offers a previously unidentified insight into potential (auto)-regulatory activity of the incretin system within the context of vascular endothelial cell biology.

Independent of the results of clinical trials designed to evaluate the potential athero-protective effects of incretin based therapeutics, including liraglutide; evidence of molecular interactions with therapeutic potential between biological systems once not appreciated to be connected represents a fascinating advance in our understanding and appreciation of the broader implications of the scientific evaluation of biological based therapeutics.

\section{References}

1. Kim W, Egan JM. The role of incretins in glucose homeostasis and diabetes treatment. Pharmacol Rev. 2008;60:470-512.

2. Drab SR. Incretin-based therapies for type 2 diabetes mellitus: current status and future prospects. Pharmacotherapy. 2010;30: 609-24.

3. Nyström T, Gutniak MK, Zhang Q, et al. Effects of glucagon like peptide on endothelial function in type 2 diabetes patients with stable coronary artery disease. Am J Physiol Endocrinol Metab. 2004;287:E1209-15.

4. Liu HB, Hu YS, Simpson RW, et al. Glucagon-like peptide-1 attenuates tumour necrosis factor-alpha-mediated induction of plasminogen activator inhibitor-1 expression. J Endocrinol. 2008;196:57-65.

5. Liu HB, Dear AE, Knudsen LB, et al. A long-acting glucagon-like peptide-1 analogue attenuates induction of plasminogen activator inhibitor type-1 and vascular adhesion molecules. J Endocrinol. 2009;201:59-66.

6. Golpon HA, Puechner A, Welte T, et al. Vasorelaxant effect of glucagon-like peptide-(7-36) amide and amylin on the pulmonary circulation of the rat. Regul Pept. 2001;102:81-6.

7. Yu M, Moreno C, Hoagland KM, et al. Antihypertensive effect of glucagon-like peptide 1 in Dahl salt sensitive rats. J Hypertens. 2003;21:1125-35.
8. Gaspari T, Liu H, Welungoda I, et al. A GLP-1 receptor agonist liraglutide inhibits endothelial cell dysfunction and vascular adhesion molecule expression in an ApoE-/- mouse model. Diab Vasc Dis Res. 2011;8:117-24.

9. Nagashima M, Watanabe T, Terasaki M, et al. Native incretins prevent the development of atherosclerotic lesions in apolipoprotein E knockout mice. Diabetologia. 2011;54:2649-59.

10. Arakawa M, Mita T, Azuma K, et al. Inhibition of monocyte adhesion to endothelial cells and attenuation of atherosclerotic lesion by a glucagon-like peptide-1 receptor agonist, exendin- 4 . Diabetes. 2010;59:1030-7.

11. Matsui T, Nishino Y, Takeuchi M, et al. Vildagliptin blocks vascular injury in thoracic aorta of diabetic rats by suppressing advanced glycation end product-receptor axis. Pharmacol Res. 2011;63:383-8.

12. Ta NN, Schuyler CA, Li Y. DPP-4 (CD26) inhibitor alogliptin inhibits atherosclerosis in diabetic apolipoprotein E-deficient mice. J Cardiovasc Pharmacol. 2011;58:157-66.

13. Puri R, Kataoka Y, Uno K, et al. The distinctive nature of atherosclerotic vascular disease in diabetes: pathophysiological and morphological insights. Curr Diab Rep. 2012;12:280-5.

14. Ryan GJ, Hardy Y. Liraglutide: once-daily GLP-1 agonist for the treatment of type 2 diabetes. J Clin Pharm Ther. 2011;36:260-74.

15. Bode B. An overview of the pharmacokinetics, efficacy and safety of liraglutide. Diabetes Res Clin Pract. 2012;97:27-42.

16. Kela R, Davies MJ. Treatment evaluation of liraglutide in type 2 diabetes. Expert Opin Biol Ther. 2012;12:1551-6.

17. Lorber D. GLP-1 receptor agonists: effects on cardiovascular risk reduction. Cardiovasc Ther. 2012. [Epub ahead of print].

18. Gaspari T, Welungoda I, Widdop RE, et al. The GLP-1 receptor agonist liraglutide inhibits progression of vascular disease via effects on atherogenesis, plaque stability and endothelial function in an apoE-/- mouse model. Diab Vasc Dis Res. 2013. In press.

19. LEADER ${ }^{\circledR}$ trial. http://clinicaltrials.gov/ct2/show/NCT01179048

20. Panjwani N, Mulvihill EE, Longuet C, et al. GLP-1 receptor activation indirectly reduces hepatic lipid accumulation but does not attenuate development of atherosclerosis in diabetic male ApoE-/- mice. Endocrinology. 2013;154:127-39.

21. Dai Y, Mehta JL, Chen M. Glucagon-like peptide-1 receptor agonist liraglutide inhibits endothelin-1 in endothelial cell by repressing nuclear factor-kappa B activation. Cardiovasc Drugs Ther. 2013;27:this issue.

22. Malm-Erjefält M, Bjørnsdottir I, Vanggaard J, et al. Metabolism and excretion of the once-daily human glucagon-like peptide-1 analog liraglutide in healthy male subjects and it's in vitro degradation by dipeptidyl peptidase IV and neutral endopeptidase. Drug Metab Dispos. 2010;38:1944-53.

23. Hattori Y, Jojima T, Tomizawa A, et al. A glucagon-like peptide-1 (GLP-1) analogue, liraglutide, upregulates nitric oxide production and exerts anti-inflammatory action in endothelial cells. Diabetologia. 2010;53:2256-63. 